\title{
Computerized Ultrasound Risk Evaluation (CURE) System: Development of Combined Transmission and Reflection Ultrasound with New Reconstruction Algorithms for Breast Imaging
}

P. J. Littrup, N. Duric, S. Azevedo, D. Chambers, J. V. U.S. Department of Energy Candy, S. Johnson, G. Auner, J. Rather, E. T. Holsapple

This article was submitted to $26^{\text {th }}$ International Acoustical Imaging Symposium, Ontario, Canada, September 9-13, 2001

September 7, 2001 


\section{DISCLAIMER}

This document was prepared as an account of work sponsored by an agency of the United States Government. Neither the United States Government nor the University of California nor any of their employees, makes any warranty, express or implied, or assumes any legal liability or responsibility for the accuracy, completeness, or usefulness of any information, apparatus, product, or process disclosed, or represents that its use would not infringe privately owned rights. Reference herein to any specific commercial product, process, or service by trade name, trademark, manufacturer, or otherwise, does not necessarily constitute or imply its endorsement, recommendation, or favoring by the United States Government or the University of California. The views and opinions of authors expressed herein do not necessarily state or reflect those of the United States Government or the University of California, and shall not be used for advertising or product endorsement purposes.

This is a preprint of a paper intended for publication in a journal or proceedings. Since changes may be made before publication, this preprint is made available with the understanding that it will not be cited or reproduced without the permission of the author.

This work was performed under the auspices of the United States Department of Energy by the University of California, Lawrence Livermore National Laboratory under contract No. W-7405-Eng-48.

This report has been reproduced directly from the best available copy.

Available electronically at http://www.doc.gov/bridge

Available for a processing fee to U.S. Department of Energy

And its contractors in paper from

U.S. Department of Energy

Office of Scientific and Technical Information

P.O. Box 62

Oak Ridge, TN 37831-0062

Telephone: (865) 576-8401

Facsimile: (865) 576-5728

E-mail: reports@adonis.osti.gov

Available for the sale to the public from

U.S. Department of Commerce

National Technical Information Service 5285 Port Royal Road

Springfield, VA 22161

Telephone: (800) 553-6847

Facsimile: (703) 605-6900

E-mail: orders@ntis.fedworld.gov

Online ordering: http://www.ntis.gov/ordering.htm

OR

Lawrence Livermore National Laboratory

Technical Information Department's Digital Library

http://www.llnl.gov/tid/Library.html 


\section{Computerized Ultrasound Risk Evaluation (CURE) System: Development of Combined Transmission and Reflection Ultrasound with New Reconstruction Algorithms for Breast Imaging}

Peter J. Littrup, Neb Duric, Stephen Azevedo, David Chambers, James V. Candy; Stephen Johnson, Gregory Auner, John Rather and Earle T. Holsapple*

\section{INTRODUCTION}

Our Computerized Ultrasound Risk Evaluation (CURE) system has been developed to the engineering prototype stage and generated unique data sets of both transmission and reflection ultrasound (US). This paper will help define the clinical underpinnings of the developmental process and interpret the imaging results from a similar perspective. The CURE project was designed to incorporate numerous diagnostic parameters to improve upon two major areas of early breast cancer detection. CURE may provide improved tissue characterization of breast masses and reliable detection of abnormal microcalcifications found in some breast cancers and ductal carcinoma in situ (DCIS). Current breast US is limited to mass evaluation, whereas mammography also detects and guides biopsy of malignant calcifications. Screening with CURE remains a distant goal, but improved follow-up of mammographic abnormalities may represent a feasible breakthrough.

Improved tissue characterization could result in reduction of the estimated one million benign biopsies each year in the United States (2), costing up to several billion dollars (3). Most breast calcifications are benign and comprise $\sim 80 \%$ of stereotactic biopsies guided by mammography (4). Ultrasound has the capability of finding some

\footnotetext{
* Peter Littrup, from the Departments of Radiology, Wayne State University, Detroit MI and Karmanos Cancer Institute, 110 East Warren Ave, Detroit MI 48201. Neb Duric, Department of Physics and Astronomy, University of New Mexico, Albuquerque, NM 87131. Steve Azevedo, David Chambers, James Candy, Lawrence Livermore National Laboratory. Stephen Johnson, Techni-Scan Inc, Salt Lake City, Utah. Greg Auner, Dept. of Electrical and Computer Engineering, Wayne State University, Detroit MI. John Rather, Departments of Physics, Wayne State University and Karmanos Cancer Institute, 110 East Warren Ave, Detroit MI. Earle Holsapple, Karmanos Cancer Institute, Detroit, MI, 48201.
} 
groups of calcifications (5-7), but further improvements in resolution should also address tissue characterization to define the soft tissue filling of ducts by DCIS. In this manner, CURE may be able to more accurately identify the malignant calcifications associated with progression of DCIS or early cancers.

Currently, high-resolution US images of the breast are performed in the reflection mode at higher frequencies, which also limits depth of penetration. Reconstruction of reflection ultrasound images relies upon acoustic impedance differences in the tissue and includes only direct backscatter of the ultrasound signal. Resolution and tissue contrast of current US continues to improve with denser transducer arrays and image processing, but the operator dependent nature of using a moveable transducer head remains a significant problem for thorough coverage of the entire breast. We have therefore undertaken the development of a whole breast (i.e., including axillary tail) system, with improved resolution and tissue characterization abilities. The extensive ultrasound physics considerations, engineering, materials process development and subsequent algorithm reconstruction are beyond the scope of this initial paper. The proprietary nature of these processes will be forthcoming as the intellectual property is fully secured. We will focus here on the imaging outcomes as they apply to eventual expansion into clinical use.

\section{MATERIALS AND METHODS}

The initial technical goal of the CURE program was to provide sub-millimeter resolution and quantitative tissue characterization using both reflection and transmission US. The initial phase had extensive computer requirements to confirm the technical feasibility by first modeling the complex scattering properties of human breast tissue. Success of these tissue scattering assumptions then required confirmation in a prototype that could test new complex algorithms. Algorithm development focused on improved localization, signal processing and depiction of quantitative US parameters. The Karmanos Cancer Institute (KCI) contracted with the Lawrence Livermore National Laboratory (LLNL) to test these concepts.

The ability to propagate sound through a phantom and receive transmitted and reflected data is far simpler than the complex tissue interactions within a human breast. Prior to engineering construction of the test scanner, computer simulation of the sound propagation and scattering within the human breast was performed. Using data from the Visible Human Project (8), a three dimensional digital model of the breast sound properties were reconstructed. Each pixel of the digitized anatomic sections was identified according to its gross histology. Fat, fibroglandular tissue, ducts, vascular and lymphatic pixels were separately assigned reported values for sound speed, index of refraction, density and absorption $(9,10)$. Computer modeling of US propagation and scattering through breast tissues served as the first proof-of-principle and verified the possibility of obtaining an improved diagnostic data set for reconstruction of high resolution and contrast images. Greater details of this portion of the project will follow in later publications as its clinical and technical merits are further defined.

A laboratory prototype scanner was designed to emphasize flexibility, simplicity of construction, and reliability of data acquisition in support of the design of a clinical prototype. This simple scanner consists of two transducers (a transmitter and receiver) independently rotating in a circular tank allowing both reflected and transmitted data 
acquisition from $0.5-5.0 \mathrm{MHz}$. The planetary scanner emulates a high-density circular array of ultrasound transducers. The stable configuration of the scanner provides robust ultrasound data sets that are ideally suited for assessing the function and stability of a variety of new reconstruction algorithms.

Producing quantitative ultrasound images requires the ability to accurately model the physics for the medium in which the US pulse travels. The properties of sound speed, attenuation and reflectivity were extracted and localized based on different assumptions about the physics of ultrasound propagation listed below:

Reflection: Reflection waves can be imaged separately to produce a two-fold gain in resolution over transmission data. However, the quantitative information of sound speed and attenuation are sacrificed. With current B-scan technology, it is also assumed that US pulses travel in straight lines and are directly transmitted back to the transducer head. Our full aperture tomography (FAT) algorithm allows circumferential processing of reflection and transmission data. An extension of reflection mode imaging, or so-called "B-scans" currently used in medical ultrasound, FAT produces images that highlight scattering sources in the insonified object. It assumes the wave scattering is isotropic and sound speed in the medium is known or calculable. The method is analogous to synthetic aperture radar (SAR) or delay-and-sum beam forming techniques (11). FAT models the object to be imaged as a collection of point scatterers. A linearizing assumption is made that acoustic energy scattered by each individual point object does not scatter off other point scatterers. This assumption of ignoring multiple scattering allows one to formulate the imaging solution for a single point scatterer independent of all other point scatterers. Then, the solution involving all point scatters becomes the linear superposition of the result from each individual point scatterer. The scattered signal from a given point in the object is sampled around the aperture (circular or paddle geometry) scanned by the receiving transducer. Assuming the point scatters isotropically, it effectively becomes a secondary point source. Thus, the goal of the imaging method is to determine the location of the point source given the data collected around the aperture.

Diffraction Physics; A quick rendering of the tissue can be obtained by approximating the US pulse interaction with the tissue and then only considering weak scattering. This approximation speeds up algorithms because the equations become much simpler. However, in regions of strong scattering, the approximation fails, resulting in strong artifacts. The diffraction tomography algorithm used in this study (DT) is built upon the work by Devaney (12) and Andre (17) and others. DT produces quantitative sound parameter images when the scattered field is sufficiently sampled relative to a wavelength. This technique includes the mathematics of diffraction using Born or Rytov models, but does not include strong scattering models. The DT method we use is performed largely in the Fourier domain with a final transform into the spatial image domain. Like FAT, DT is a single step process, with no iteration, so it can be performed reasonably quickly. It produces quantitative images of sound speed and attenuation.

Full Wave Propagation: The most thorough reconstruction involves solving the wave equation. These methods are sometimes called full wave or inverse scattering algorithms. They take the measured field (i.e. the scattered field) and, from its properties, deduce the acoustic parameters of the propagating medium. These algorithms are more accurate at the expense of greater processing time. The method used in this study was developed by Johnson, et al, (13) and is described therein.

Proprietary algorithms, covering all three cases, were utilized in order to construct images of the insonified phantoms. Two basic phantoms were used to 
approximate mass margins and punctate resolution (e.g., cyst and microcalcifications). The "cyst" phantom was a $4 \mathrm{~cm}$ diameter cylinder containing uniform US-scattering agar and two smaller cylinders, separated from each other and imbedded in the agar. The smaller cylinders were one-centimeter in diameter and contained a water-alcohol mixture with a lower sound speed than the surrounding agar. In this manner, the cylinders mimicked the slower sound speed in fluid filled cysts in a low contrast setting. A second phantom (henceforth the "calcification" phantom) consisted of a 4-cm diameter cylinder containing ten, $0.2-\mathrm{mm}$ diameter strings embedded through the cylinder at a fixed radius. The strings were grouped into five pairs with separations ranging from two to five $\mathrm{mm}$ within each pair. The final phantom consisted of a normal cadaveric breast placed in formalin and sealed within a plastic container.

The two phantoms and cadaveric breast specimen were scanned by the CURE prototype at a frequency of $1.5 \mathrm{MHz}$ using 2 microsecond pulses. A total of 1280 receiver positions and 320 transmitter positions were used, corresponding to lambda/ 4 and lambda spacing, respectively. The relatively low frequency of $1.5 \mathrm{MHz}$ was used in order to better penetrate the phantoms and broaden the beam angle while retaining sub-mm spatial resolution. Each data set represents a 2-D slice through the phantom. The phantoms were also scanned with a clinical reflection ultrasound unit (GE Logiq 600) and a clinical CT scanner. All CURE scans were performed at 10 millimeter slice thickness to generate multiple tomographic images per phantom. Each slice required a two-hour scan time to emulate a fully populated array within the prototype. The CT scans were performed at $1.25 \mathrm{~mm}$ slice thickness.

\section{RESULTS}

The standard US B-scan image (Figure 1A) shows the ability of high-frequency US to detect $0.2 \mathrm{~mm}$ strong reflectors in a uniform, low scattering medium. However, the 6-11 $\mathrm{MHz}$ bandwidth shows resolution degradation of line pair crispness and separation in the far field, even in this ideal medium. Figure 1B shows the excellent point definition and separation of the CURE scanner using a full aperture reflection algorithm at $1.5 \mathrm{MHz}$. Figure $1 \mathrm{C}$ is a transmission image using a full wave algorithm. These images show adequate point separation and ability to quantify sub-millimeter regions, despite the inherently worse resolution of transmission US.

The standard B-scan reflection ultrasound image (Figure 2A) shows the characteristic "cystic", or anechoic, appearance of the fluid cylinders. These have a sharp leading edge, but limited lateral and posterior wall definition. Figure 2B shows a CURE reflection image at $1.5 \mathrm{MHz}$ reconstructed with a full aperture tomography (FAT) reflection algorithm. Despite the low frequency, the margins of the "cysts" are crisply defined throughout their circumference and show improved detail. One cylinder contained an involuted segment, whereas the other cylinder had an exophytic linear region corresponding to an adjacent crack in the agar. These cylinder contours were not as well defined by the standard B-scan image due to its dependence on transducer position and assumption of only 180 degree backscatter. Figure $2 \mathrm{C}$ and $2 \mathrm{D}$ are transmission images which confirm the lower attenuation and sound speed (i.e., darker centers) of the cylinder contents. The attenuation image made by our diffraction algorithm (Figure $2 \mathrm{C}$ ) still shows more of the cylinder contour detail than standard US, 
but some contour artifacts are produced at the margins of strong scattering. The sound speed image (Figure 2D) was reconstructed using a full wave algorithm and demonstrates lower sound speed within the cylinder than the adjacent agar. These subtle attenuation and sound speed differences are of comparable magnitude to the differences between cyst fluid and adjacent normal breast tissue.

Figure $3 \mathrm{~A}$ is an X-ray $\mathrm{CT}$ image generated with $1.25 \mathrm{~mm}$ slice thickness through the normal breast specimen showing predominantly fatty tissue with only thin internal fibrous bands. Figure 3B is a standard B-scan ultrasound image of the specimen showing three important facets of a complex scattering medium with high-frequency reflection US: 1) imaging is operator dependent and limited to the field of view of the linear array transducer width; 2) The far field becomes severely degraded by the compounding effect of complex scattering in a fatty specimen when relying upon reflection mode from only one source:receiver position; and 3) point resolution in the near field also is limited to the fibrous bands when they are perpendicular to the US beam and form specular reflectors (i.e., internal architecture of fibrous bands are not seen parallel to the beam). Figure 3C is the CURE image obtained at $1.5 \mathrm{MHz}$ in which the reflection and transmission data are simply overlayed and contains no additional post-processing to enhance tissue detail. The CURE image shows the overall specimen configuration with excellent morphologic comparison to the CT scan. There is also no loss of signal or resolution seen in the near or far field. Despite the low frequency of the CURE image, the fine architectural detail of internal fibrous bands is similar to the CT image (the latter was made at a thinner slice thickness and resultant high radiation exposure). Tissue characterization of ultrasound parameters was not directly correlated with any histologic section, but the background sound speed pattern matches the overall fatty appearance on the CT scan. We acknowledge that further tissue correlation studies are warranted and were not the focus of this feasibility study.

\section{DISCUSSION}

The feasibility of submillimeter resolution ultrasound with potential tissue characterization by sound parameters has been demonstrated for the engineering prototype phase of the CURE program. These goals were also confirmed in a complex scattering medium of cadaveric human breast tissue, substantiating the predictions from the initial computer simulation of wave propagation from the transformed Visible Woman data set (8). The potential benefits for clinical breast imaging from angleindependent scanning are improved tumor margin visualization, while maintaining excellent resolution and thorough penetration.

Breast tissue characterization by current reflection US may be best represented by the success of the 'Stavros/Colorado' criteria for mass margin evaluation (14). The $98 \%$ accuracy obtained for defining a benign mass rested upon the criterion of an ovoid mass with well-defined margins. This was sufficient for ATL, Inc. to obtain separate Food and Drug Administration approval for characterizing benign breast masses with their high-resolution transducer in 1996 (15). However, little clinical progress has been noted for additional US tissue characterization to improve breast tissue diagnosis. The CURE images (Figures 2B, 2C, 2D) suggest that mass margin definition may be greatly expanded when evaluated from multiple locations, and perhaps in 3 dimensions. CURE identification of clear mass margins therefore does not suffer from the need for tumor 
capsules $(6,14)$ to be discretely identified as specular reflectors, nor from incomplete lateral margins from diffraction artifacts. Our "cyst" phantom clearly demonstrated improved margin evaluation for both reflection and transmission algorithms. The possibility for three-dimensional evaluation of all aspects of tumor mass may hold great promise for improved tissue characterization by simple morphology.

The "calcification" phantom demonstrates the excellent resolution of high frequency B-scan reflection US in a uniform scattering medium (Figure 1A), but also shows the opposing effect of degraded penetration in the far field. CURE images were most notable for the exquisite point resolution of the FAT reflection algorithm (Figure 1B) and the discrete localization of sound speed for the transmission images (Figure 1C). The performance of the FAT algorithm at $1.5 \mathrm{MHz}$ may remove resolution concerns at much deeper tissue depths than seen with high frequency B-scan US (Figure 3B). Improved penetration without loss of resolution may also explain the excellent morphologic correlation of the CURE cadaveric breast image (Figure $3 \mathrm{C}$ ) with the CT scan. Better structural detail, tissue characterization and deep penetration are demonstrated relative to the B-scan images. Yet, the CURE specimen image was not optimized by post-processing and may have additional partial averaging degradation of resolution since the CURE slice thickness was $10 \mathrm{~mm}$, compared to the $1.25 \mathrm{~mm}$ thick CT slice. The improved resolution and tissue characterization possibilities seen in the CURE cadaveric image continue to offer promise of routine detection of not only malignant calcifications, but also their association with tissue-filled breast ducts. Further work is needed in both freshly excised breast specimens and patients with subsequent biopsy confirmation.

The current study is limited in scope and does not provide the convincing histopathologic imaging correlation desired when introducing a new technology. We fully acknowledge that we are not introducing a new clinical imaging system at this time. We are, however, presenting the clinical rationale and an encouraging pilot study, which continue to drive our pursuit of this challenging physics, computer, materials processing and engineering program. Previous work in transmission US $(13,17)$ showed similar promise but languished due to lack of computer processing ability and associated algorithm development. The promise of our work is based on the production of robust and reproducible experimental data, the utilization of advances in computer technology and renewed algorithm development. We plan to report on the continuing development of this project in future publications.

\section{ACKNOWLEDGMENTS}

The authors would like to acknowledge the support of several individuals in this work. They are Michael Berggren of Techni-Scan, Inc., for the use of their phantoms and for their reconstructions, Dr. John Boone at U. C. Davis Medical center for the X-ray CT images and Alex Babkin and Robert Duncan (University of New Mexico) for their help with the image processing. 


\section{REFERENCES}

1. Woolf SH. The accuracy and effectiveness of routine population screening with mammography, prostatespecific antigen, and prenatal ultrasound: a review of published scientific evidence. Int J Technol Assess Health Care 2001; 17(3):275-304.

2. Liberman L. Clinical management issues in percutaneous core breast biopsy. Radiol Clin North Am. 2000; 38:791-807.

3. Secker-Walker RH, Vacek PM, Hooper GJ, Plante DA, Detsky AS. Screening for breast cancer: time, travel, and out-of-pocket expenses. J Natl Cancer Inst 1999, 91:702-8.

4. Makoske T, Preletz R, Riley L, Fogarty K, Swank M, Cochrane P, Blisard D. Long-term outcomes of stereotactic breast biopsies. Am Surg. 2000, 66:1104-8.

5. Cleverley JR, Jackson AR, Bateman AC. Pre-operative localization of breast microcalcification using highfrequency ultrasound. Clin Radiol. 1997; 52:924-6.

6. Skaane P. Ultrasonography as adjunct to mammography in the evaluation of breast tumors. Acta Radiol Suppl. 1999; 420:1-47.

7. Teh WL, Wilson AR, Evans AJ, Burrell H, Pinder SE, Ellis IO. Ultrasound guided core biopsy of suspicious mammographic calcifications using high frequency and power Doppler ultrasound. Clin Radiol. 2000; 55:390-4.

8. Visible Human Project, National Library of Medicine, Bethesda, Maryland, http://www.nlm.nih.gov/research/visible/visible human.html.

9. Goss, S.A., Johnston, R.L. and Dunn, F. Comprehensive compilation of empirical ultrasonic properties of mammalian tissues. J Acoust Soc AM 1978; 64: 423-457

10. Edmonds, P.D., Mortensen, C.L., Hill, J.R., Holland, S.K., Jensen, J.F., Schattner, P. and Valdes, A.D. Ultrasound tissue characterization of breast biopsy specimens. Ultrasound Imaging 1991; 13:162-185.

11. Fitch, J. P., Synthetic Aperture Radar, Springer-Verlag, 1988.

12. A. J. Devaney. A filtered backpropagation algorithm for diffraction tomography. Ultrasonic Imaging, 4(4):336-350, October 1982.

13. S. A. Johnson and M. L. Tracy. Inverse scattering solutions by a sinc basis, multiple source, moment method. Part I: Theory, Ultrasonic Imaging, 5:361-375, 1983.

14. Stavros AT, Thickman D, Rapp CL, Dennis MA, Parker SH, Sisney GA. Solid breast nodules: use of sonography to distinguish between benign and malignant lesions. Radiology. 1995;196:123-34.

15. 61 Federal Register 60712-60713 (1996). (http://www.rsna.org/REG/research/regulatory/wfprfcexamples.html)

16. Schreiman JS, Gisvold JJ, Greenleaf JF, Bahn RC. Ultrasound transmission computed tomography of the breast. Radiology 1984; 150:523-30.

17. André MP, Janee HS, Martin PJ, Otto GP, Spivey BA, Palmer DA. High-speed data acquisition in a diffraction tomography system employing large-scale toroidal arrays. International Journal of Imaging Systems and Technology 1997; 8:137-147.

(a)

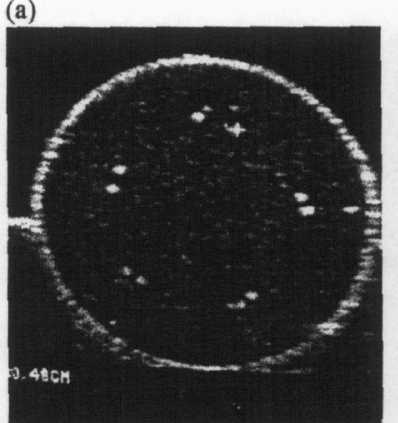

(b)

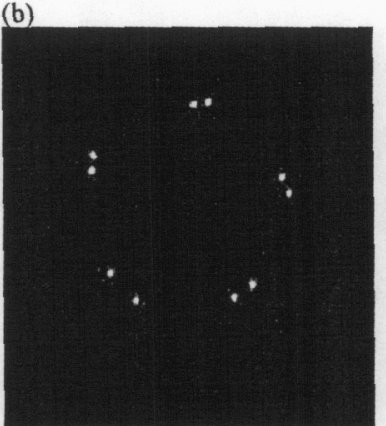

(c)

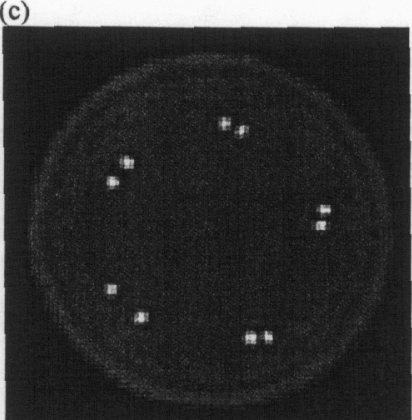

Figure 1. (a) B-Scan image of the "calcification" phantom. (b) Full aperture reflection image. (c) Sound speed reconstruction using the full wave algorithm. 
(a)

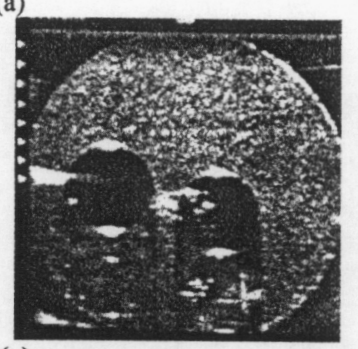

(c)

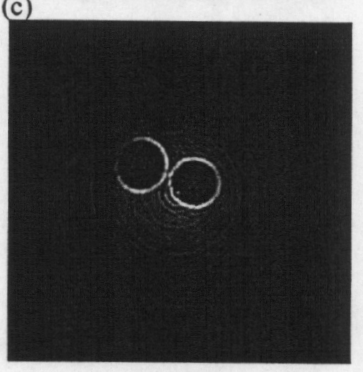

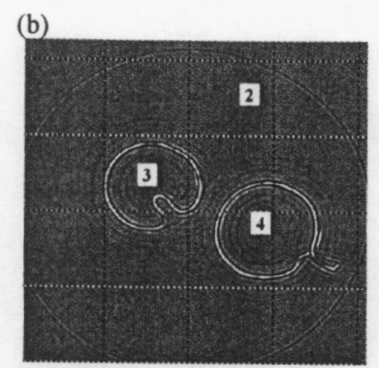

(d)

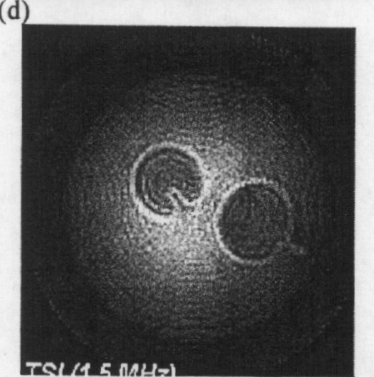

Figure 2. (a) The B-scan image of the "cyst" phantom. (b) The full aperture reflection image of the cyst phantom. (c) The attenuation image from the diffraction algorithm. (d) The sound speed image using the full wave algorithm.
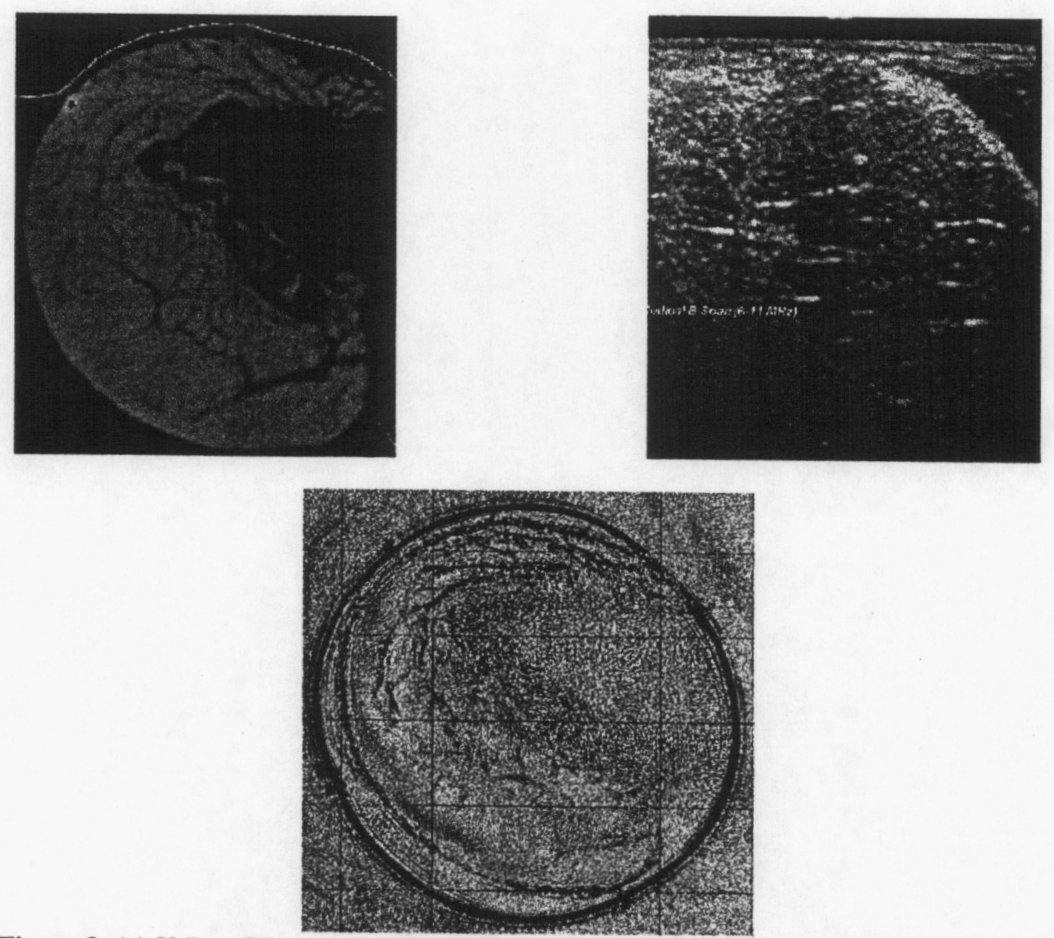

Figure 3. (a) X-Ray CT scan of cadaveric breast. (b) B-Scan of a portion of the breast. (c) Reflection image superimposed on a sound-speed reconstruction. 